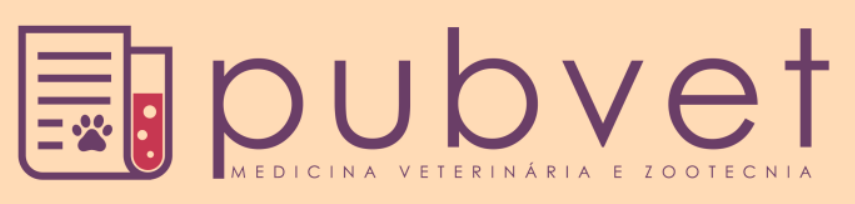

https://doi.org/10.31533/pubvet.v12n9a173.1-9

\title{
Qualidade e rotulagem de ovos comercializados no município de Valença - RJ
}

\author{
Lucas Barros Corrêa Netto ${ }^{1 *}{ }^{\bullet}$, Lívia Melo da Silva1 ${ }^{\bullet}$, Marta Maria Braga Baptista \\ Soares Xavier ${ }^{20}$
}

${ }^{I}$ Estudante de Medicina Veterinária, Fundação Educacional Dom Andre Arcoverde Centro De Ensino Superior De Valença

${ }^{2}$ Prof ${ }^{a}$. Dr. ${ }^{a}$ Faculdade De Medicina Veterinária, Fundação Educacional Dom Andre Arcoverde Centro De Ensino Superior De Valença

*Autor para correspondência, E-mail: lucasbcnetto@hotmail.com

\begin{abstract}
RESUMO. $O$ ovo é considerado um alimento completo por suas características nutricionais, devido a isso é consumido por todas as classes sociais. As condições de armazenamento são fundamentais para manter as qualidades nutricionais dos ovos para isso é necessário que seja feita avaliação do produto desde o processo de saída das granjas até a chegada no comercio de onde irá para a mesa do consumidor final. Esse trabalho teve como objetivo avaliar os ovos que são comercializados no município de Valença - RJ e verificar se estão aptos para irem a mesa do consumidor, para isso foram adquiridas 10 cartelas de ovo contendo 12 ovos em cada e foram observadas as condições higiênico sanitárias e como os ovos eram mantidos nos estabelecimentos. Os 12 atributos avaliados foram classificados em conformidade e não conformidade, tendo como conclusão que os estabelecimentos não estão comercializando os ovos de acordo com a legislação.
\end{abstract}

Palavras chave: ovos, higiene alimentar, inspeção de ovos

\section{Quality and labeling of eggs marketed in the city of Valença - RJ}

\begin{abstract}
The egg is considered a complete food by its nutritional characteristics, and because of it is consumed by all social classes. The storage conditions are fundamental to maintain the nutritional qualities of the eggs. For this it is necessary an evaluation of the product from the process of leaving the farms to the arrival in the market from where it will go to the final consumer's table. The objective of this work was to evaluate the eggs that are marketed in the city of Valença - RJ and verify if they are ready to go to the consumer's table. For this purpose, 10 cartons containing 12 eggs were obtained, and the hygienic sanitary conditions and how the eggs were kept in establishments were observed. The 12 evaluated attributes were classified in conformity and nonconformity, having as conclusion that the establishments are not marketing the eggs according to the legislation.
\end{abstract}

Keywords: Eggs, food hygiene, eggs inspection

\section{Calidad y etiquetado de huevos comercializados en el municipio de Valencia - RJ}

RESUMEN. El huevo es considerado un alimento completo por sus características nutricionales, debido a eso es consumido por todas las clases sociales. Las condiciones de almacenamiento son fundamentales para mantener las calidades nutricionales de los huevos para ello es necesario que se haga una evaluación del producto desde el proceso de salida de las granjas hasta la llegada al comercio de donde irá a la mesa del consumidor final. Este trabajo tuvo como objetivo evaluar los huevos que se comercializan en el municipio de 
Valença - RJ y verificar si son aptos para ir a la mesa del consumidor, para ello se adquirieron 10 cartones de huevo conteniendo 12 huevos en cada y se observaron las condiciones higiénicas sanitarias y como los huevos eran mantenidos en los establecimientos. Los 12 atributos evaluados fueron clasificados en consecuencia y no conformidad, teniendo como conclusión que los establecimientos no están comercializando los huevos de acuerdo con la legislación.

Palabras clave: huevos, higiene alimentaria, inspección de huevos

\section{Introdução}

O ovo é consumido por todas as classes sociais devido ser um alimento com baixo custo e excelente fonte de vários nutrientes, tais como folato, riboflavina, selênio, colina e vitaminas $\mathrm{A}, \mathrm{D}, \mathrm{E}, \mathrm{K}, \mathrm{B} 12$, contém sais minerais (ferro, fósforo, cálcio, magnésio, sódio, potássio, cloro, iodo, manganês, enxofre, cobre, zinco), proteína de alta qualidade e lipídios, que tornam biodisponíveis importantes nutrientes como luteína e zeaxantina, associados com a prevenção da degeneração muscular, além de fonte de gorduras saturadas e colesterol (Santos et al., 2013).

De acordo com Alcântra (2012), o consumo de ovos e utilização de suas vantagens nutricionais pela população estão associados à qualidade do produto oferecido ao consumidor, que é determinada pelo conjunto de características que podem influenciar na aceitabilidade do produto no mercado. Tais características são valorizadas pelos consumidores, que estão cada vez mais exigentes, sendo percebidas pelos atributos sensoriais, nutricionais, tecnológicos, sanitária e ausência de resíduos químicos.

Para os produtores a qualidade dos ovos está relacionada ao peso, aparência da casca, tais como ausência de sujidades, defeitos, trincas e manchas de sangue já para os consumidores o prazo de validade e características sensórias, como odor e a cor da gema são essenciais para atestar a qualidade do ovo (Alleoni \& Antunes, 2001).

A qualidade insatisfatória dos ovos pode acarretar prejuízos econômicos e a saúde do consumidor. Uma das maiores preocupações da comercialização dos ovos in natura é a contaminação por microrganismos. A maioria dos ovos apresentam baixa contaminação no momento da postura, a contaminação ocorre geralmente após a ovoposição, por provável contato com as fezes das aves no momento da postura, a contaminação através de rachaduras microscópicas da casca ou através poros após o processo de lavagem. (Alcântra, 2012; AragonAlegro et al., 2005).
Segundo Andrade et al. (2004), a contaminação dos ovos, seja na casca ou no conteúdo é o principal fator de qualidade que deve ser rigorosamente inspecionado na tentativa de garantir segurança alimentar do consumidor, já que estudos demonstram que há alta incidência de contaminação de ovos no mercado por Salmonella spp.

A contaminação da casca do ovo é importante para a determinação de seu tempo de vida na prateleira e para a segurança alimentar dos consumidores. Para que se consiga manter o potencial nutritivo do ovo, algumas técnicas de preservação como a lavagem da casca, o armazenamento em baixas temperaturas, a pasteurização, podem ser empregadas na tentativa de diminuir problemas decorrentes da contaminação por microrganismos patógenos deteriorantes (Aregon-Alegro et al., 2012).

Práticas higiênico sanitárias devem ser adotadas em todas as etapas de produção com o intuito de evitar a contaminação dos ovos para garantir ao consumidor um alimento seguro.

A rotulagem é de grande importância, sendo a identidade do produto, pois permite ao consumidor ter acesso a diversas informações, como os valores nutricionais, classe ao que o ovo pertence, sua cor, assim como aos parâmetros sobre a qualidade e segurança do seu consumo, como o prazo de validade, atuação correta quanto a conservação e consumo do produto. As informações devem ser sempre esclarecedoras, verdadeiras e completas (Moraes et al., 2007)

Diante do exposto, esse trabalho teve por objetivo avaliar a qualidade, rotulagem dos ovos comercializados no município de Valença/RJ, segundo a legislação sanitária vigente.

\section{Revisão de literatura}

\section{Produção e consumo de ovos}

A maior parte dos ovos comercializados no Brasil é produzida com alta tecnologia por poedeiras comerciais obtidas através de cruzamentos de 
várias linhagens genéticas, resultando em aves altamente produtivas (Santos et al., 2009).

Segundo o relatório anual da Associação Brasileira de Proteína Animal (ABPA, 2017), o consumo per capita de unidades por ano teve um aumento significativo nos últimos anos, indo de 148 unidades/ano em 2010 para 190 em 2016. Esse aumento se explica por ser uma proteína de origem animal de fácil acesso e baixo custo (ABPA, 2017).

Atualmente $99,57 \%$ da produção de ovos é destinada ao mercado interno e $0,43 \%$ é destinado para a importação, desse valor $84 \%$ é exportado in natura e $16 \%$ é industrializado sendo a África o principal importador (ABPA, 2017).

Conforme Alcântra (2012), o consumo de ovos e a utilização de suas vantagens nutricionais pela população, está associado a qualidade do produto oferecido ao consumidor, que é influenciado por um conjunto de características que podem influenciar a aceitabilidade do produto no mercado. Um conjunto de características influenciam a qualidade dos ovos e a aceitabilidade dos consumidores, sendo diversos aspectos determinantes como internos e externos. Os aspectos internos são as características relativas ao albúmen, gema, cor, odor, sabor. Os aspectos externos são a cor da casca, tamanho, presença de sujidades (Mendes, 2010).

Segundo a ANVISA (2009), as granjas produtoras de ovos comerciais estão sujeitas a fiscalização do Serviço de Inspeção Federal e da Agencia Nacional de Vigilância Sanitária, portanto, adotam medidas específicas de produção e comercialização de ovos, visando manter a qualidade do produto final.

\section{Valor nutricional do ovo}

A composição do ovo varia de acordo com uma série de fatores intrínsecos que incluem: espécie, manejo, nutrição idade, genética, instalações e sanidade. O ovo é atribuído como um alimento de alto valor biológico, pois as proteínas encontradas em sua composição é fonte de vários aminoácidos, é considerado um alimento completo com várias fontes de nutrientes, tais como selênio, vitaminas (A, D, E, K e B12), colina, folato, riboflavina, além de sais minerais, proteínas de alta qualidade e lipídeos, gorduras saturadas e colesterol. Por esta grande quantidade de nutrientes ( $\underline{\text { Santos et }}$ al., 2013).
Algumas proteínas do ovo possuem propriedades anti-nutricionais, quando não são desnaturadas, devido a isso, não se deve consumir ovo cru em quantidades elevadas. Entre essas propriedades incluem inibidores das enzimas digestivas ovomucóide, ovoinibidor e a ovotransferrina (Seibel, 2005).

Conforme citado por Quadros et al. (2011), sendo considerado um dos alimentos naturais mais equilibrado em nutrientes, baixa concentração calórica e completo, ovo é composto por proteínas, ácidos graxos, gorduras, minerais, lipídeos, glicídios e vitaminas. E é considerado uma das fontes protéicas de origem animal mais baratas e acessíveis encontradas no mercado (Tabela 1).

Tabela 1. Valores nutricionais do ovo.

\begin{tabular}{|c|c|c|}
\hline Nutrientes & Unidade & Quantidade \\
\hline Energia & Kcal & 143 \\
\hline Proteína & $\mathrm{g}$ & 12,56 \\
\hline Carboidrato & $\mathrm{g}$ & 0,72 \\
\hline Gordura total & $\mathrm{g}$ & 9,51 \\
\hline G. Monoinssaturada & $\mathrm{g}$ & 3,6 \\
\hline G. Polinssaturada & $\mathrm{g}$ & 1,9 \\
\hline Gordura saturada & g & 3,1 \\
\hline Gordura trans & g & 0 \\
\hline Colesterol & $\mathrm{mg}$ & 372 \\
\hline Colina & $\mathrm{mg}$ & 293 \\
\hline Riboflavina (B2) & $\mathrm{mg}$ & 0,45 \\
\hline Cianocobalina (B12) & $\mu \mathrm{g}$ & 0,89 \\
\hline Ácido Fólico & $\mu \mathrm{g}$ & 0 \\
\hline Vit. D & $\mathrm{IU}$ & 82 \\
\hline Vit. A & $\mathrm{IU}$ & 540 \\
\hline Pirodoxina (B6) & $\mathrm{mg}$ & 0,17 \\
\hline Tiamina (B1) & $\mathrm{mg}$ & 0,04 \\
\hline Vit. E & $\mathrm{mg}$ & 1,05 \\
\hline Selenium & $\mu \mathrm{g}$ & 30,7 \\
\hline Fósforo & $\mathrm{mg}$ & 198 \\
\hline Ferro & $\mathrm{mg}$ & 1,75 \\
\hline Zinco & $\mathrm{mg}$ & 1,29 \\
\hline Cálcio & $\mathrm{mg}$ & 56 \\
\hline Sódio & $\mathrm{mg}$ & 142 \\
\hline Potássio & $\mathrm{mg}$ & 138 \\
\hline Magnésio & $\mathrm{mg}$ & 12 \\
\hline
\end{tabular}

Como descrito por Almeida et al. (2017) um dos maiores fatores de preocupação em relação ao consumo de ovos é a saúde pública, pois quando consumido in natura, armazenado de 
forma inadequada, ingerido fora da validade, dentre outros fatores, pode causar intoxicações gastrointestinais, contaminação por Salmonella sp., por Staphylococcus aureus, ou Coliformes (abrange todas as bactérias na forma de bacilos gram negativos, não esporogênicos, aeróbios ou anaeróbios facultativos, capazes de fermentar a lactose com produção de gás, dentro de 24 a 48 horas em uma temperatura de $35^{\circ} \mathrm{C}$.)

\section{Armazenamento e Temperatura:}

$\mathrm{O}$ ovo começa a perder as qualidades internas no momento após a postura, caso não sejam tomadas medidas adequadas para a sua conservação. A perda da qualidade ocorre de forma continua ao longo do tempo e pode ser agravada por diferentes fatores (Barbosa et al., 2008).

De acordo com Alcântara (2012), o tempo e a temperatura de armazenamento do ovo influenciam na conservação dos ovos, as medidas de conservação podem prolongar o tempo de prateleira desse produto.

Segundo Lopes et al., (2012), ovos mantidos sobre refrigeração até 25 dias após a postura apresentam boas condições para o consumo, embora no Brasil não haja legislação que exija a refrigeração dos ovos comerciais, bem como, não há prazo de validade máximo estabelecido. Geralmente os ovos são mantidos em temperatura ambiente desde a produção até sua distribuição final. Segundo Lana et al. (2017), os ovos armazenados em locais refrigerados ganham mais tempo de prateleira para até 30 dias mantendo a boa qualidade interna.

O ideal seria que os ovos fossem mantidos refrigerados desde o momento de saída das granjas e continuassem assim até o consumo já que a refrigeração protege a qualidade interna dos ovos (Pires et al., 2015). Lopes et al. (2012), lembra que em alguns estabelecimentos os ovos comercializados são colocados próximos as verduras e freezer, com o objetivo de diminuir a temperatura, deixando-os um pouco abaixo da temperatura ambiente.

\section{Microrganismos vinculados pelo consumo de ovo causadores de doenças alimentares}

Segundo Pires (2013), a população tende a acreditar que o consumo de ovos vendidos em feiras e diretamente de granjas sejam mais saudáveis do que os ovos comercializados nas redes de supermercados, o que leva ao descaso com os cuidados em relação à sanidade da produção e armazenamento, esquecendo das possíveis contaminações e da garantia de qualidade.

Segundo Rêgo et al. (2012), o ovo é rico em nutrientes de alta digestibilidade e por isso exige cuidados para não se tornarem meio de crescimento para microrganismos e chegarem deteriorados ao consumidor.

O envoltório natural do ovo é a casca, que tem como a função principal protegê-lo contra injurias mecânicas e entrada de microorganismos. A superfície da casca contém pequenos poros que permitem troca gasosa entre o ovo e o ambiente, assim ocorrendo a entrada de oxigênio e a saída de gás carbônico, porém essa porosidade também facilita a entrada de microorganismos que pode contaminar o produto em condições normais, pois o ovo possui uma barreira de proteção natural, chamada cutícula que tem duração de quatro dias, após esse período há redução na proteção, desencadeando ressecamento da casca e rachaduras (Medeiros \& Alves, 2014).

Para diminuir a contaminação dos ovos pela casca, o Manual de Segurança e Qualidade para a Avicultura de Postura (EMBRAPA, 2004), determina que de acordo com a legislação internacional que os ovos sejam lavados com o devido cuidado, com detergentes especial e sanitizado, usa-se óleo mineral natural que não contenha odor, nem sabor e que seja inofensivo a saúde humana para repor a proteção da casca do ovo.

Alguns autores discutem sobre os efeitos de desinfecção da lavagem sobre a casca do ovo, no entanto Aragon-Alegro et al. (2005), defende que os ovos submetidos a lavagem reduzosriscos decorrentes da contaminação por microorganismos patógenos deteriorantes

Como descrito por Almeida et al.(2017) um dos maiores fatores de preocupação em relação ao consumo de ovos é a saúde pública, pois quando consumido in natura, armazenado de forma inadequada, ingerido fora da validade, dentre outros fatores, pode causar intoxicações gastrointestinais, contaminação por Salmonella sp. ou por Staphylococcus aureus, ou Coliformes (abrange todas as bactérias na forma de bacilos gram negativos, não esporogênicos, aeróbios ou anaeróbios facultativos, capazes de fermentar a 
lactose com produção de gás, dentro de 24 a 48 horas em uma temperatura de $35^{\circ} \mathrm{C}$.)

O consumo de alimentos a base de ovos crus ou mal cozidos podem colocar a saúde do consumidor em risco, dentre os patógenos mais prováveis de causar toxicoinfecção está a Salmonella. Segundo Barancelli et al.(2012), a Salmonella é um dos principais patógenos envolvidos em casos de surtos de doenças transmitidas por alimentos e sua complexa epidemiologia dificulta seu controle no ambiente e na produção avícola o que representa uma constante preocupação em termos de segurança alimentar, tanto na produção quanto no processamento e consumo de ovos.

A contaminação do ovo por salmonela pode acontecer de duas maneiras: contaminação vertical, que ocorre internamente durante sua formação no trato reprodutivo infectado da ave, ou pela contaminação horizontal, que é a introdução de bactérias através dos microporos da casca do ovo (Barancelli et al., 2012).

\section{Classificação dos ovos:}

Conforme a Portaria $\mathrm{n}^{\circ} 1$, do MAPA, os ovos de galinha, tanto os destinados para o mercado interno como para o mercado externo são classificados em extra, especial, primeira qualidade, segunda qualidade, terceira qualidade e fabrico que são os ovos que não estão apropriados para a comercialização inteiros mas que estão em boas condições de consumo, podendo ser usados para processamento.

Os ovos podem ser classificados quanto a qualidade de cinco maneiras: A, B, C, D e E (Tabela 2), quanto ao peso como: tipo 1 jumbo (peso mínimo de $66 \mathrm{~g}$ por unidade), tipo 2 extra (peso mínimo 60 a $65 \mathrm{~g}$ por unidade), tipo 3 grande (peso mínimo 55 a $59 \mathrm{~g}$ por unidade), tipo 4 médio (peso mínimo de 50 a $54 \mathrm{~g}$ por unidade), tipo 5 pequeno (peso mínimo 45 a $49 \mathrm{~g}$ por unidade) e tipo 6 industrial que apresenta o peso mínimo abaixo de $45 \mathrm{~g}$ por unidade (Tabela 3 ). (Oliveira \& Oliveira, 2013).

\section{Legislação e rotulagem}

A rotulagem é um elemento importante para assegurar que a população tenha acesso a informações sobre os alimentos, suas características, formas de conservação e informações necessárias para a manutenção da saúde e prevenção de doenças. Existem legislações para a elaboração dos rótulos, que precisam ser seguidas onde deverão conter informações obrigatórias como o prazo de validade, ingredientes, lote e a rotulagem nutricional. Os ovos e alguns outros alimentos como o palmito possuem legislação específica sobre a rotulagem adequada.

Tabela 2. Classificação de ovos segundo Resolução CIPOA nº 5/91.

\begin{tabular}{|c|c|c|c|c|}
\hline & Casca & Câmara de Ar & Albúmen & Gema \\
\hline A & $\begin{array}{l}\text { Limpa } \\
\text { Íntegra } \\
\text { Sem deformação }\end{array}$ & $\begin{array}{l}\text { Fixa } \\
\text { Máx. 4mm }\end{array}$ & $\begin{array}{l}\text { Límpido } \\
\text { Transparente } \\
\text { Consistente } \\
\text { Calazas íntegras }\end{array}$ & $\begin{array}{l}\text { Translúcida } \\
\text { Consistente } \\
\text { Centralizada } \\
\text { S/ desenvolvimento do germe }\end{array}$ \\
\hline B & $\begin{array}{l}\text { Limpa } \\
\text { Íntegra } \\
\text { Ligeira deformação } \\
\text { Discretamente manchada }\end{array}$ & $\begin{array}{l}\text { Fixa } \\
\text { Máx. } 6 \mathrm{~mm}\end{array}$ & $\begin{array}{l}\text { Límpido } \\
\text { Transparente } \\
\text { Reativamente Consistente } \\
\text { Calazas íntegras }\end{array}$ & $\begin{array}{l}\text { Ligeiramente descentralizada e } \\
\text { deformada } \\
\text { Contorno definido } \\
\text { S/ desenvolvimento do germe }\end{array}$ \\
\hline $\mathrm{C}$ & $\begin{array}{l}\text { Limpa } \\
\text { Íntegra } \\
\text { Defeitos de textura e } \\
\text { contorno } \\
\text { Manchada }\end{array}$ & $\begin{array}{l}\text { Solta } \\
\text { Máx. } 10 \mathrm{~mm}\end{array}$ & $\begin{array}{l}\text { Ligeiramente turvo } \\
\text { Relativamente Consistente } \\
\text { Calazas íntegras }\end{array}$ & $\begin{array}{l}\text { descentralizada e deformada } \\
\text { Contorno definido } \\
\text { S/ desenvolvimento do germe }\end{array}$ \\
\hline $\mathrm{D}$ & \multicolumn{4}{|c|}{ Ovo sujo - Não quebra, com sujeira ou material externo aderente, manchas moderadas. } \\
\hline $\mathrm{E}$ & \multicolumn{4}{|c|}{$\begin{array}{l}\text { Ovo trincado - Com casca quebrada ou rachada, mas cujas as membranas da casca estejam intactas e cujo } \\
\text { conteúdo não vaze. }\end{array}$} \\
\hline
\end{tabular}

Fonte: Brasil, 1991. 
Tabela 3. Classificação de ovos segundo o peso.

\begin{tabular}{lllllll}
\hline & Jumbo & Extra & Grande & Médio & Pequeno & Industrial \\
\hline \multirow{2}{*}{ Peso } & Mín. 66g & Mín. 60g & Mín. 55g & Mín. 50g & Mín. 45g & $<45 \mathrm{~g}$ \\
& & Máx. 65g & Máx. 59g & Máx. 54g & Máx. 49g & \\
\hline
\end{tabular}

Fonte: Oliveira \& Oliveira, (2013).

A normativa RDC 35 da ANVISA, 2009 em seu artigo $5^{\circ}$, determina além dos dizeres exigidos para alimentos que conste na rotulagem dos ovos as seguintes expressões: "O consumo deste alimento cru ou mal cozido pode causar danos à saúde", "Manter os ovos preferencialmente refrigerados". Essas expressões devem ser declaradas em destaque, de forma legível.

\section{Material e Métodos}

Foi realizado um estudo descritivo observacional, no qual foram feitas coletas de amostras para a pesquisa nos supermercados do município de Valença/RJ.

Foram compradas 10 cartelas de ovos com 12 ovos em cada. As cartelas de ovos comercializadas pertenciam a diferentes distribuidores e foram classificadas de A a J para não identificar os locais de origem.

Dentre os supermercados visitados, foram observadas as condições higiênico sanitárias dos estabelecimentos, as condições que os ovos eram mantidos nas prateleiras e se eram mantidos sobre refrigeração.

Os rótulos das embalagens foram avaliados conforme a recomendações da legislação vigente no país, decreto $n^{\circ} 56.585$ de 20 de julho de 1965 . Observando se nela constavam as informações básicas como: tipo de ovo, grupo, classe, data de classificação, prazo de validade e identificação do lote.

A adequação dos atributos foram avaliados em conformidade (C) e não conformidade (NC) de acordo com a legislação vigente. As conformidades referiam-se ao peso dos ovos, ausência de sujidades, ovos sem trincas, embalagem, cumprimento do declarado na rotulagem e prazo de validade.

\section{Resultados e Discussão}

As tabelas4 e $\underline{5}$ apresentam os resultados obtidos referentes a conformidade e não conformidade dos parâmetros avaliados nas embalagens de ovos.
Com relação aos resultados obtidos, apenas $10 \%$ atendia a todos os requisitos da legislação vigente e estava de acordo para o consumo. $30 \%$ das amostras estavam com o prazo de validade vencido e ainda assim eram comercializadas.

Todas as marcas respeitaram a data da embalagem, como também a informação de "não contém glúten" para os grupos alérgicos, orientações para o armazenamento dos ovos e a informação de que o ovo cru ou mal cozido pode causar riscos à saúde do consumidor. A identificação e a classificação dos ovos também foram respeitadas por todas as marcas.

Apenas $50 \%$ das amostras estavam livres de sujidades, as marcas que não estavam em conformação apresentavam ovos com sujeiras, mofo e presença de fezes, em $30 \%$ dos casos que as amostras apresentavam sujidades também haviam ovos trincados, com unidades presas na embalagem e odor pútrido muito forte. Observouse que as amostras que continham mais sujidades eram as que estavam em embalagens que continham alguma abertura, constatando-se que as cartelas fechadas protegem mais os ovos de sujidades e do risco de trincas.

Uma grande vantagem nos ovos está na sua casca, que confere proteção além de ser uma embalagem natural de um produto de alto valor biológico. A qualidade externa do ovo está ligada a qualidade de sua casca, independentemente da cor, a casca deve estar limpa, íntegra e sem deformações. As deformações no formato do ovo prejudicam o visual e podem causar problemas sanitários ao animal. Um dos maiores defeitos na casca são as trincas, que facilitam a entrada de microrganismos facilitando a perda da qualidade (Sarcinelli et al., 2007).

A visualização da casca além de ser um fator importante para a venda do produto, pois os consumidores tendem a escolher por ovos com a casca com um aspecto mais agradável, esta relacionada com a qualidade do ovo. Considerada a embalagem do ovo, a casca deve estar sempre limpa, íntegra e ainda sem deformações, pois cascas resistentes protegem a parte interna (Sarcinelli et al., 2007). 
Tabela 4. Classificação em conformidade e não conformidade dos parâmetros avaliados dos produtos.

\begin{tabular}{lcccccccccc}
\hline Marca & A & B & C & D & E & F & G & H & I & J \\
\hline Supermercado & C & 1 & 1 & 1 & 2 & 2 & 2 & 3 & 3 & 3 \\
Grupo & Branco & Branco & Cor & Branco & branco & cor & cor & branco & cor & branco \\
Identificação & C & C & C & C & C & C & C & C & C & C \\
Classificação & C & C & C & C & C & C & C & C & C & C \\
Tipificação & C & C & C & NC & C & C & C & NC & NC & C \\
Selo & C & C & C & C & C & C & C & C & C & C \\
\hline
\end{tabular}

Tabela 5.Classificação em conformidade e não conformidade dos parâmetros avaliados dos produtos.

\begin{tabular}{|c|c|c|c|c|c|c|c|c|c|c|}
\hline Marca & $\mathbf{A}$ & B & $\mathbf{C}$ & D & $\mathbf{E}$ & $\mathbf{F}$ & $\mathbf{G}$ & $\mathbf{H}$ & I & $\mathbf{J}$ \\
\hline Sujidade & $\mathrm{C}$ & $\mathrm{NC}$ & $\mathrm{C}$ & $\mathrm{C}$ & $\mathrm{C}$ & $\mathrm{NC}$ & $\mathrm{C}$ & $\mathrm{NC}$ & $\mathrm{NC}$ & $\mathrm{NC}$ \\
\hline Ovos trincados & $\mathrm{C}$ & $\mathrm{C}$ & $\mathrm{C}$ & $\mathrm{C}$ & $\mathrm{C}$ & $\mathrm{NC}$ & $\mathrm{C}$ & $\mathrm{NC}$ & $\mathrm{C}$ & $\mathrm{NC}$ \\
\hline Informação nutricional & $\mathrm{C}$ & $\mathrm{C}$ & $\mathrm{NC}$ & $\mathrm{NC}$ & $\mathrm{NC}$ & $\mathrm{C}$ & $\mathrm{C}$ & $\mathrm{NC}$ & $\mathrm{NC}$ & $\mathrm{C}$ \\
\hline Instruções de conservação & $\mathrm{C}$ & $\mathrm{NC}$ & $\mathrm{NC}$ & $\mathrm{NC}$ & $\mathrm{NC}$ & $\mathrm{C}$ & $\mathrm{C}$ & $\mathrm{C}$ & $\mathrm{C}$ & $\mathrm{C}$ \\
\hline Data da embalagem & $\mathrm{C}$ & $\mathrm{C}$ & $\mathrm{C}$ & $\mathrm{C}$ & $\mathrm{C}$ & $\mathrm{C}$ & $\mathrm{C}$ & $\mathrm{C}$ & $\mathrm{C}$ & $\mathrm{C}$ \\
\hline Data de validade & $\mathrm{NC}$ & $\mathrm{C}$ & $\mathrm{C}$ & $\mathrm{NC}$ & $\mathrm{C}$ & $\mathrm{C}$ & $\mathrm{C}$ & $\mathrm{C}$ & $\mathrm{NC}$ & $\mathrm{C}$ \\
\hline "Não contém glúten" & $\mathrm{C}$ & $\mathrm{C}$ & $\mathrm{C}$ & $\mathrm{C}$ & $\mathrm{C}$ & $\mathrm{C}$ & $\mathrm{C}$ & $\mathrm{C}$ & $\mathrm{C}$ & $\mathrm{C}$ \\
\hline
\end{tabular}

Em relação as informações nutricionais $50 \%$ não continha na embalagem e $30 \%$ estava em desacordo com as normas, que estabelecem que cada categoria de ovo deve seguir uma tabela nutricional específica de acordo com o tipo do ovo.

Nos supermercados onde as amostras foram adquiridas, observou-se que os ovos para venda eram expostos próximos a áreas com frízer o que poderia evitar a exposição ao calor que diminuiria a vida útil de prateleira.

No Brasil por não ser obrigatório a refrigeração, os ovos comerciais são acondicionados desde o momento da postura até a distribuição final em temperaturas ambientes, sendo em alguns casos refrigerados apenas nas casas dos consumidores.

\section{Conclusão}

O trabalho demonstra a importância de inspeção sanitária nos estabelecimentos que comercializam ovos no município de Valença.

Com os resultados obtidos, demonstra que os estabelecimentos não estão comercializando os ovos de acordo com a legislação, comercializando produtos fora do prazo de validade e fora dos padrões ideais de armazenamento em locais refrigerados, também existe a necessidade de inspecionar as empresas distribuidoras que embalam os ovos com a rotulagem nutricional errada levando o consumidor ao erro.

As inadequações do comercio e da rotulagem dos ovos podem acarretar problemas a saúde pública, sugerindo-se que além melhorar os programas de Boas Práticas de Fabricação e Análise de Perigos e Pontos Críticos de Controle - APPCC na indústria. É fundamental o papel de manipuladores e consumidores na diminuição dos riscos de salmoneloses, principalmente em relação a contaminações cruzadas, pois por maiores que sejam os esforços, não é possível garantir a inocuidade de um produto in natura como o ovo, portanto, é imprescindível, a execução de ações educativas sobre práticas seguras de compra, armazenamento e manipulação de ovos. Tendo em vista a importância epidemiológica de Salmonella para as DTAs e a problemática de sua ocorrência na cadeia de produção avícola e em ovos.

\section{Referências bibliográficas}

ABPA, Associação Brasileira de Proteína Animal. 2017. Relatoria Anual 2017 (p. 110-125). 
Alcântara, Juliana Bonifácio de. 2012. Qualidade físico química de ovos comerciais: Avaliação e manutenção da qualidade. UFG.

Alleoni, A. C. C. \& Antunes, A. J. 2001. Unidade Haugh como medida da qualidade de ovos de galinha armazenados sob refrigeração. Scientia Agrícola, 58(4), 681-685.

Almeida, C. A., Friebel, D., Rossa, R. B. \& Gelinski, J. M. L. 2017. Avaliação das Condições Higiênico-Sanitárias da Casca e Gema de Ovos In Natura. Anuário pesquisa e extensão Unoesc Videira, 2.

Andrade, M. A., Café, M. B., Jayme, V. S., Rocha, P. T., Leandro, N. S. M. \& Stringhini, J. H. 2004. Avaliação da qualidade bacteriológica de ovos de galinha comercializados em Goiânia. Goiás. Brasil. Ciência Animal Brasileira, 5(4), 221-228.

ANVISA (Brasil). Resolução RDC $n^{\circ} .35$, de junho de 2009. Aprova o Regulamento Técnico que estabelece instruções de conservação e consumo na rotulagem de ovos. Diário Oficial da União, 18 de junho de 2009;

ANVISA, Agencia Nacional de Vigilância Sanitária. 2009. Resolução RDC $\mathrm{n}^{\circ} .35$, de junho de 2009. Aprova o Regulamento Técnico que estabelece instruções de conservação e consumo na rotulagem de ovos. Diário Oficial da União, 18 de junho de 2009.

Aragon-Alegro, L. C., Souza, K. L. O., Sobrinho, P. S. C., Landgraf, M. \& Destro, M. T. 2005. Avaliação da qualidade microbiológica de ovo integral pasteurizado produzido com e sem a etapa de lavagem no processamento. Ciência e Tecnologia de Alimentos, 25(3), 618-622.

Barancelli, G. V., Martin, J. G. P. \& Porto, E. 2012. Salmonella em ovos: relação entre produção e consumo seguro. Segurança Alimentar e Nutricional, 19(2), 73-82.

Barbosa, A. A., Sakomura, N. K., Mendonça, M. O., Freitas, E. R. \& Fernandes, J. B. K. 2008. Qualidade de ovos comerciais provenientes de poedeiras comerciais armazenados sob diferentes tempos e condições de ambientes. Arquivos de Veterinária, 24(2), 127-133.

BRASIL. Ministério da Agricultura e do Abastecimento. Secretaria de Defesa Agropecuária. Divisão de Inspeção de Carnes e Derivados - DICAR. Resolução 005 de 19/11/91 da Coordenação de Inspeção de Produtos de Origem Animal - CIPOA. 1991.
BRASIL. Ministério da Agricultura, Pecuária e Abastecimento. Decreto 56.585, 25 jul. 1965.

EMBRAPA, Empresa Brasileira de Pesquisa Agropecuária. 2004. Manual de segurança e qualidade para a avicultura de postura. Segurança e qualidade dos alimentos. Embrapa Informação Tecnológica, BR.

Lana, R. S. V., Lana, G. R. Q., Salvador, E. L., Lana, Â. M. Q., Cunha, F. S. A. \& Marinho, A. L. 2017 Qualidade de ovos de poedeiras comerciais armazenados em diferentes temperaturas e períodos de estocagem. Revista Brasileira de Saúde e Produção Animal, 18(1), 140-151.

Lopes, L. L. R. A., Silva, Y. L., Nunes, R. V., Takahashi, S. E. \& Mori, C. 2012. Influência do tempo e das condições de armazenamento na qualidade de ovos comerciais. Revista Cientifica Eletrônica de Medicina Veterinária, 9(18), 1-15.

Medeiros, F. M. \& Alves, M. G. M. 2014. Qualidade de ovos comerciais. Revista Eletrônica Nutri Time, 11(4), 3515-3524.

Mendes, F. R. 2010. Qualidade física, química e microbiológica de ovos lavados armazenados sob duas temperaturas e experimentalmente contaminados com Pseudomonas aeruginosa. Universidade Federal de Goiás, Goiânia, 72f.

Moraes,I. A., Mano,S. \& Baptist, R. F. 2007. Análise da rotulagem de ovos comercializados na cidadedo Rio de Janeiro - Brasil. Revista brasileira de Ciência veterinária, 14(1), 7-11

Oliveira, B. L. \& Oliveira, D. D. 2013. Qualidade e tecnologia de ovos. Ufla, Lavras, Minas Gerais, Brasil.

Pires, M. F., Pires, S. F., Andrade, C. L., Carvalho, D. P., Barbosa, A. F. C. \& Marques, M. R. 2015. Fatores que afetam a qualidade dos ovos de poedeiras comerciais. Revista Eletrônica Nutritime, 12,(6), 4379-4385.

Pires, M.F., 2013. Aspectos De Qualidade FísicoQuímica E Microbiológica De Ovos Comerciais. 2013. Universidade Federal De Goiás Escola De Veterinária E Zootecnia Graduação Em Zootecnia, Goiânia, 39p.

Quadros, D. G., Jesus, T. R., Kanematsu, C. H., Sá, A. M., Silva, G. A. V., Silva, A, L, R. \& Andrade, A. P. 2011. Qualidade de ovos de galinha comercializados em Barreiras, BA, estocados em diferentes condições de temperatura. Revista Acadêmica, Ciências Agrárias e Ambientais, 9(4), 363-369. 
Rêgo, I. O. P., Cançado, S. V., Figueiredo, T. C., Menezes, L. D. M., Oliveira, D. D., Lima, A. L., Esser, L. R. 2012. Influência do período de armazenamento na qualidade do ovo integral pasteurizado refrigerado. Arquivo Brasileiro de Medicina Veterinária e Zootecnia, 64(3), 735-742.

Santos, M. S. V., Espíndola, G. B., Lôbo, R. N. B., Freitas, E. R., Guerra, J. L. \& Santos, A. B. E. 2009. Efeito da temperatura e estocagem em ovos. Ciência e Tecnologia de Alimentos campinas, 29(3): 513-517.

Santos, R. D., Gagliardi, A. C. M., Xavier, H. T., Magnoni, C. D., Cassani, R., Lottenberg, A. M. P., Casella Filho, A., \& Ramos, S. 2013. I Diretriz sobre o consumo de Gorduras e Saúde Cardiovascular. Arquivos Brasileiros de Cardiologia, 100(1Supl.3), 1-40.
Sarcinelli, M. F., Venturini, K. S. \& Silva, L. C. 2007. Características dos ovos. Universidade federal do Espírito Santos, Espirito Santo, BR.

Seibel, N. F. 2005. Transformações bioquímicas durante o processamento do ovo. In: SouzaSoares, L. A. \& Siewerdt, F. Aves e ovos (p. 7790). UFPEL, Pelotas, Rio Grande do Sul, Brasil.

Recebido: 25 Junho. 2018.

Aprovado:7 Agosto. 2018

Publicado:13 Setembro. 2018

Licenciamento: Este artigo é publicado na modalidade Acesso Aberto sob a licença Creative Commons Atribuição 4.0 (CC-BY 4.0), a qual permite uso irrestrito, distribuição, reprodução em qualquer meio, desde que o autor e a fonte sejam devidamente creditados. 